\title{
Mismeasure of secondary sexual traits: an example with horn growth in the Iberian ibex
}

\author{
M. Sarasa ${ }^{1 \star}$, R. C. Soriguer ${ }^{1,2}$, J. -E. Granados ${ }^{1,3}$, N. Casajus ${ }^{4}$ \& J. M. Pérez ${ }^{1,5}$ \\ 1 Grupo Biología de las Especies Cinegéticas y Plagas, Sevilla, Spain \\ 2 Estación Biológica de Doñana, Sevilla, Spain \\ 3 Espacio Natural Sierra Nevada, Pinos Genil, Granada, Spain \\ 4 Chaire de Recherche du Canada en Conservation des Écosystèmes Nordiques et Centre d'Études Nordiques, Université du Québec à Rimouski, \\ Rimouski, QC, Canada \\ 5 Departamento de Biología Animal, Biología Vegetal y Ecología, Universidad de Jaén, Jaén, Spain
}

\author{
Keywords \\ bovids; Caprinae; methodological bias; \\ phenotypic characterization; sexual \\ selection; ungulates; weapons. \\ Correspondence \\ *Present address: Mathieu Sarasa, 10, \\ place des Oustalots, F-64400 \\ Oloron-Sainte-Marie, France. \\ Email: mathieusar@hotmail.com
}

\begin{abstract}
Monitoring programmes and studies focused on secondary sexual characters (SSCs) depend on the accuracy of measurements. However, methods of measurements of SSC, such as horns of ungulates, vary throughout the literature. Thus, the accuracy of horn growth measurements as proxies of true horn growth and the comparability of results inferred from different horn growth measurements may be questionable. We used the horns of Iberian ibex Capra pyrenaica to compare horn growth measurements and to analyse reliability with true horn growth. Our results reveal that measurements used in previous studies differed substantially from true horn growth and volume estimated as a barrel appeared as the best proxy of annular segments of horns in the Iberian ibex. Horn growth measurements are not necessarily mutually comparable, just as classical measurements are not necessarily representative of true horn growth. We discuss the wider implications of these results and suggest that biological processes linked to horns of ungulates should be reappraised using improved and accurate measurements because horn growth pattern is a key factor in sustainable management and conservation plans of ungulate species around the world.
\end{abstract}

\section{Introduction}

Secondary sexual characters (SSCs) may have evolved due to the pressures of sexual selection and life-history trade-offs (Stearns, 1992; Emlen, 2008). Such traits are more or less honest signals of individual quality (Malo et al., 2005; Lailvaux, Reaney \& Backwell, 2009; Bergeron et al., 2010) that often lead to uneven reproductive success and fitness between individuals within populations (Coltman et al., 2002; Garant, Dodson \& Bernatchez, 2003; Preston et al., 2003; Bartoš \& Bahbouh, 2006). As a result, SSCs are considered to be a key factors in the ecology of species and thus are of great interest to behavioural and evolutionary ecologists (Emlen, 2008; Cornwallis \& Uller, 2010).

Furthermore, certain obvious SSCs may draw the attention of laypeople as some of these impressive traits may well be the main characteristic of an iconic animal species. For example, the ornamented male of the Betta fighting fish Betta splendens is a very popular species in the aquarium trade (Dennis \& Aldhous, 2004), while the trophy score of bovids is positively related to the prices paid by recreational hunters (Johnson et al., 2010). As a result, the SSCs of animal species are subject to commercial and potentially selective pressures (Mysterud, 
2011) and are therefore of great significance to managers and conservationists alike.

Monitoring programmes and studies focused on SSCs depend on a basic question: how can secondary sexual traits be measured accurately? Just as the image perceived by the eye depends on the prism that is used, the accuracy of monitoring programmes and studies focused on SSCs will be determined by the estimated parameters that are used. Several good examples of this notion can be found in the literature. While initial studies on colour signalling in birds assumed that birds see colour patterns as humans do (Bennett, Cuthill \& Norris, 1994), nowadays the wide potential of signals that are unseen by the human eye (e.g. ultraviolet signals) are highlighted and taken into account (Bennett et al., 1994, 1996). Likewise, today, a more refined understanding of acoustic signalling is possible because ultrasonic sounds can now be taken into account (Croll et al., 2002; Holy \& Guo, 2005).

In ungulates, much attention has been paid to weapons such as horns and antlers, which are usually well developed and even act as a differential trait in males (Emlen, 2008). However, methods of measurements of horns and antlers vary throughout the literature. The most widespread way of measuring these weapons is their length, probably due to the influ- 
ence of hunter's criteria when scoring their trophies. However, such weapons have complex tri-dimensional structures, and so in cervids, several authors have considered the whole ramification of the antlers and have used other calculations (e.g. the number of antler tines, number of antler tops and weight) that take into account such complexity (Bartoš \& Bahbouh, 2006; Bartoš, Bahbouh \& Vach, 2007). In Caprinae, horns are not ramified, but their tri-dimensional growth has encouraged a number of authors to consider horn growth by estimating the volume of annular segments (Loehr et al., 2007, 2010). However, even the tri-dimensional estimates of horn growth may be prone to non-negligible inaccuracies when compared with true volumes (Hoefs \& Nowlan, 1997) and, thus, the accuracy of both horn growth measurement as a proxy of true horn growth and the comparability of results inferred from different horn growth measurements may be questionable.

Nowadays, most knowledge of horn size in Caprinae is based on estimates of length rather than of volume. Mismatches can even be found in the biological interpretations derived from studies employing different types of measurements. For example, trade-offs between horn growth rates and longevity have been suggested in Dall sheep Ovis dalli on the basis of volume estimates of horn annular segments (Loehr et al., 2007); yet, on the basis of horn length measurements, horn length was found not to constrain longevity in the Alpine ibex Capra ibex (Bergeron et al., 2008). To date, it is hazardous to attribute such mismatches to either biological or methodological factors. Only a few conference proceedings have ever presented preliminary results on the comparability of measurements of horn growth (König \& Hoefs, 1984), and to date, this question is a much under-considered subject.

Here, we used the horns of Iberian ibex Capra pyrenaica (Sarasa, Alasaad \& Pérez, 2012) to compare horn growth measurements and to test the following predictions. If typical measurements (estimated length and volume) are a true representation of horn growth, very good and comparable correlations are to be expected between common measurements and the true volume and weight of annular segments (prediction 1). If common measurements are comparable proxies of true horn growth, very strong correlations are also to be expected between classical measurements themselves (prediction 2) and comparable standardized variances (SVs) are to be expected between measurements (prediction 3). Furthermore, if all measurements are good proxies of true horn growth, the biological results inferred from different horn growth measurements - such as the age-dependent pattern of horn growth in our sample - will converge. More specifically, the age at which horn growth starts to slow down should be the same for all the different horn growth measurements (prediction 4).

\section{Material and methods}

\section{Study site and population}

We studied the Iberian ibex population in the Sierra Nevada mountain range $\left(36^{\circ} 00^{\prime}-37^{\circ} 10^{\prime} \mathrm{N}, 2^{\circ} 34^{\prime}-3^{\circ} 40^{\prime} \mathrm{W}\right.$, Spain), a reference population in Iberian ibex ecology (Sarasa et al., 2010a,b, 2011a,b; Pérez et al., 2011). Our analysis was based on 260 annular segments from 36 male Iberian ibexes, culled by the Natural Space staff for management purposes (Espacio Natural de Sierra Nevada). The age of animals was determined by counting horn increments (Fandos, 1991).

\section{Laboratory procedure and measurements}

We considered several measurements taken from frozen skulls. Annular segment length was measured to the nearest $0.5 \mathrm{~mm}$ with a digital calliper along the internal crest of the horn, which is easily visible along the whole length of the horn. Also, the apical circumference, mid-annulus circumference and basal circumference of each growth segment were measured to the nearest millimetre with a flexible tape measure. Subsequently, horns [the complete structure including from the bone to the keratinized epidermis (Davis, Brakora \& Lee, 2011)] were cut into sections with a hacksaw at the horn-ring growth stop formed during each winter of the ibex's life. Estimation of the 'true volume' of each annular section was carried out using the water displacement method (König \& Hoefs, 1984) to the nearest $5 \mathrm{~mL}$ with beakers. Each annular section was weighed to the nearest $0.01 \mathrm{~g}$.

Parameters were measured twice to calculate the repeatability (r) of multiple measurements of the same annulus. All measures were performed by the first author to avoid interobserver variability.

Few authors have estimated the volume of each annular section using the conical frustum formula (Loehr et al., 2007, 2010). Thus, the volume as a conical frustum was estimated for our annular sections. We also observed that numerous annular segments were shaped like a barrel. Using the mean between apical and basal circumference, the volume as a barrel was estimated for our annular sections as well (Kepler's formula).

\section{Statistical analysis}

The first annular segment that grows from birth to the first winter in the ibex's life, which is usually disregarded because it is subjected to breaks (Bergeron et al., 2008; Loehr et al., 2010), was excluded from our analysis. Incompletely grown annuli were also excluded from analysis. The repeatability (r) of measurements and of volume estimates were calculated from the variance components derived from one-way analysis of variance (Lessells \& Boag, 1987). Measurement error as a proportion of the total within-annulus variance was also estimated $\left(\mathrm{MS}_{\mathrm{w}} / \mathrm{MS}_{\mathrm{a}} ¥ 100\right)$. In subsequent analyses, the mean of duplicate measures was considered. To identify the best proxies of horn growth in Iberian ibex, we first estimated the $\mathrm{SV}$ of measurements as the ratio of variance in a considered trait to the square of the mean of the considered trait, which represents an upper limit to the strength of directional sexual selection (Wade \& Arnold, 1980). Then, to standardize the scales of measurement, entries were transformed into standardized variables by the relationship:

$$
z_{x}=\left(v_{x}-m_{x}\right) / \sigma_{x}
$$


where $z_{x}$ is the standardized value, $v_{x}$ is the original value, and $m_{x}$ and $o_{x}$ is the mean and standard deviation of the variable, respectively. Using Spearman's correlations, we examined the relationship between annular length, annular basal circumference, estimated volumes of annular segments (calculated with the conical- and barrel-derived formulas), the true volume of annular segments and the true weight of annular segments.

Then, to compare the biological results inferred from each measurement method, we used generalized additive models (Wood, 2006) that allow non-linear relationships to be fitted between independent and dependent variables. Annular length, annular basal circumference, estimated volumes of annular segments (calculated with the conical frustra formula and with Kepler's barrel formula), the true volume of annular segments and the weight of annular segments were modelled as a function of age. The reference of each ibex was included as a random term. For each measurement, fitted values were used to estimate the age at which the horn growth rate started to decrease in our sample.

All analyses were performed using the R statistical software (R Development Core Team, 2011).

\section{Results}

The within-annulus repeatability of measurements was high (Table 1). Annular weight and volume estimated with water displacement exhibited the greatest repeatability ( $r=1$ and 0.99 , respectively), followed by basal circumference $(r=0.91)$ and volume estimated as conical frustra $(r=0.90)$. Annular length and volume estimated as a barrel also had high repeat- ability ( $r=0.79$ and 0.73 , respectively), although these values were lower than for the afore-mentioned measurements.

In terms of SV (Table 1), volumes estimated as a barrel and with water displacement and annular weight had the highest values ( $\mathrm{SV}=0.34,0.31$ and 0.32 , respectively). The SV of the volume estimated as conical frustra was almost half as high. The SV of other measurements (annuli length and circumferences) was four to five times lower than the SV of true horn growth.

Measurements of true horn growth (annulus volume measured with water displacement and annulus weight) exhibited a correlation that was very close to 1 ; nevertheless, proxies of horn growth (estimates of annulus length, circumference and volume) correlated differently with measurements of true horn growth (Table 2). Kepler's barrel formula for estimating annular volumes exhibited the highest correlation with true horn growth, followed by the estimate as conical frustra (Table 2). Length and basal circumference of annular segments exhibited the lower correlation with true horn growth (Table 2).

General additive models of measurements showed different patterns according to the measurement used (Fig. 1). Annular length suggests increasing horn growth from 2 to 5 years of age and a decreasing trend thereafter (Fig. 1a). Volume as conical frustra also suggests a bell-shaped pattern with a maximum value at 6-years old (Fig. 1c). Basal circumference exhibited an increasing trend from 2 to 9 years of age (Fig. 1b). Annular volume estimated as a barrel and measurements of true horn growth showed an increasing trend that might reach an asymptote after 7-years old (Fig. 1d-f).

Table 1 Repeatability and variance of measurements of annuli

\begin{tabular}{lrlll}
\hline & & Measurement error (\% of total within-individual & \\
Measurement & F ratio & variance) & Repeatability (r) & Standardized variance (SV) \\
\hline Length & 8.76 & 11.4 & 0.79 & 0.06 \\
Apical circumference & 8.52 & 11.7 & 0.79 & 0.08 \\
Mid-annuli circumference & 8.92 & 11.2 & 0.80 & 0.05 \\
Basal circumference & 21.52 & 4.6 & 0.91 & 0.04 \\
Volume estimated as conical frustra & 18.84 & 5.3 & 0.90 & 0.16 \\
Volume estimated as a barrel (Kepler) & 6.29 & 15.9 & 0.73 & 0.34 \\
Volume estimated with water displacement & 661.23 & 0.1 & 0.99 & 0.31 \\
Weight & - & 0 & 1.00 & 0.32 \\
\hline
\end{tabular}

Table 2 Correlation between measurements of annuli

\begin{tabular}{|c|c|c|c|c|c|}
\hline & BC & $V_{c}$ & $V_{\mathrm{b}}$ & $V$ & Weight $(W)$ \\
\hline \multirow[t]{2}{*}{ Length (L) } & $r_{s}=0.51$ & $r_{s}=0.85$ & $r_{s}=0.73$ & $r_{s}=0.74$ & $r_{\mathrm{s}}=0.73$ \\
\hline & $P<0.001$ & $P<0.001$ & $P<0.001$ & $P<0.001$ & $P<0.001$ \\
\hline \multirow[t]{2}{*}{ Basal circumference (BC) } & & $r_{s}=0.87$ & $r_{s}=0.95$ & $r_{s}=0.88$ & $\mathrm{r}_{\mathrm{s}}=0.89$ \\
\hline & & $P<0.001$ & $P<0.001$ & $P<0.001$ & $P<0.001$ \\
\hline \multirow[t]{2}{*}{ Volume estimated as conical frustra $\left(V_{c}\right)$} & & & $r_{s}=0.98$ & $r_{s}=0.95$ & $r_{s}=0.95$ \\
\hline & & & $P<0.001$ & $P<0.001$ & $P<0.001$ \\
\hline \multirow[t]{2}{*}{ Volume estimated as barrel, Kepler $\left(V_{b}\right)$} & & & & $r_{s}=0.96$ & $r_{s}=0.96$ \\
\hline & & & & $P<0.001$ & $P<0.001$ \\
\hline \multirow[t]{2}{*}{ Volume estimated with water displacement (true volume, $V$ ) } & & & & & $\mathrm{r}_{\mathrm{s}}=0.99$ \\
\hline & & & & & $P<0.001$ \\
\hline
\end{tabular}



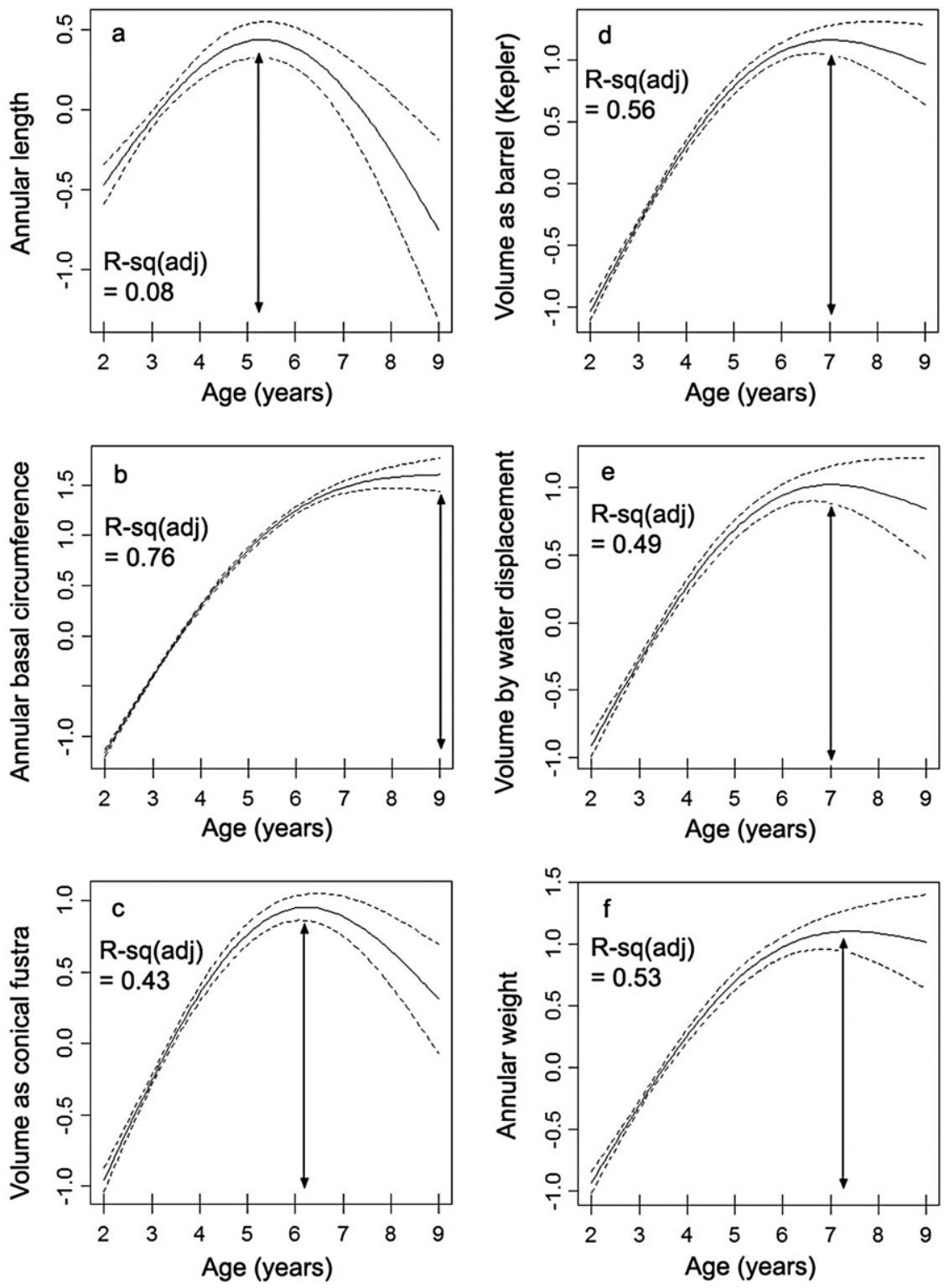

Figure 1 Relationship between annular horn growth and age according to different measurements: (a) annular length; (b) annular basal circumference; (c) volume as conical frustra; (d) volume as barrel (Kepler); (e) volume by water displacement; and $(f)$ annular weight. All measurements were centred-reduced. R$\operatorname{sq}(\operatorname{adj})=\operatorname{adjusted} R^{2}$ of the fitted model.

Almost half of the observed variability in true horn growth was explained in models as a function of age (Fig. 1e,f). Models of estimated volume (as a barrel and as conical frustra) as a function of age exhibited fits that were comparable to those of true horn growth (Fig. 1c,d). Age explained a greater proportion of variability in the basal circumference of annular sections than in true horn growth (Fig. 1b). The model of the length of annular segments as a function of age exhibited the worst fit, which was five to six times lower than those of true horn growth (Fig. 1a).

\section{Discussion}

To our knowledge, our results are the first detailed comparison of horn growth measurements that take into account several different criteria for indentifying the best proxy of true horn growth in Caprinae. It is important to note that all of the measurements used in previous studies and included in our analysis differed substantially from true horn growth. Thus, previous studies on horn growth should be considered with caution. Hunting-score indexes rank trophies on the basis of the global biometry of the horns - for instance, using total length, basal circumferences and maximal spacing between horns (Luzon et al., 2008; Hedrick, 2011) - but do not necessarily take into account the age of the animal; this fact probably blurs the biological significance of such data. Huntingscore indexes were not available in our dataset (which was based on annular segments) and other authors also have previously mentioned that these indexes do not properly reflect real horn size (König \& Hoefs, 1984). Thus, hunting-score 
indexes should also be subjected to rigorous comparisons with true horn growth prior to validating any biological interpretation derived from such data. Horns are rarely cut into sections, maybe because of their economic value as trophies (Johnson et al., 2010). Consequently, our study highlights the benefits to research and to biological study that can be derived from collaboration with public institutions such as protected natural spaces and reserves that are interested in ungulate conservation and are not subject to commercial trophyhunting pressure.

The main objective of our analysis was to confirm or discard potential differences between measurements. Our results underline the fact that estimates from different methods have weak differences in repeatability and display substantial differences in variance, in correlation with true horn growth and, above all, in biological meaning. In our sample, the volume estimated as a barrel $\left(V_{\mathrm{b}}\right)$ had comparable SV and the highest correlation with true horn growth (volume estimated with water displacement and annular weight). Also, biological results inferred from $V_{\mathrm{b}}$ - such as the age-dependent pattern of horn growth in our sample - converged with the results derived from true horn growth. Thus, volume estimated as a barrel appeared as the best proxy of true horn growth in the Iberian ibex. The repeatability of $V_{\mathrm{b}}$ was slightly lower than that of the other measurements, although further methodological improvements, such as an increase in the replicates of measurements, might improve this parameter.

The comparison between true horn growth and other measurements highlight interesting discrepancies. Correlations of annular length with volume estimated by water displacement and with annular weight were the poorest. Moreover, the age-dependent pattern of annular length disagrees with the age-dependent pattern of true horn growth. This result underlines the fact that the relationship between annular length and true horn growth changes with age. Consequently, when analysing biological processes in horn growth linked to age - for example, trade-offs and senescence (von Hardenberg et al., 2004; Bergeron et al., 2008) - the accuracy of annular length is strongly questionable, as our example clearly illustrates. Although annular length suggests that horn growth increases until 5-years old, the volume estimated by water displacement and annular weight shows that horn growth increased until at least 7-years old in our sample. This result questions allocation patterns in horn growth previously reported in ibexes (Alvarez, 1990; Fandos, 1995; von Hardenberg et al., 2004) and partially agrees with studies that suggest that senescence in ibexes only becomes evident in animals over 8-years old (Couturier, 1962; Schaller, 1977; Toїgo et al., 2007). Thus, greater knowledge of senescence and allocation in horn growth in ibexes is still required.

It is also important to note that the basal circumference of annuli had a better correlation with true horn growth than annular length. This result illustrates that the relationship between annular circumference and true horn growth becomes steadier with age than the relationship between annular length and true horn growth. Yet, the horns of ibexes are considered as 'long' weapons (Couturier, 1962) and the importance attached to their thickness is less than in the horns of wild sheep (Schaller, 1977). Consequently, further studies are still required to check whether the biological interpretations inferred from annular length in wild sheep converge with the biological results that could be inferred from accurate proxies of true horn growth.

The case of volume estimated as conical frustra $\left(V_{c}\right)$ is also very interesting. Although $V_{\mathrm{c}}$ exhibited high values of repeatability and of correlation with true horn growth, the agedependent pattern of $V_{c}$ differed from those of true horn growth. $V_{c}$ suggests that horn growth would increase until 6-years old and decrease thereafter; yet, true horn growth showed that horn growth increased until at least 7-years old in the ibex from our sample. Also, the SV of $V_{c}$ was almost half as high as that of true horn growth in our sample. Thus, although measurements might correlate well with true horn growth, this does not necessarily mean that they are good proxies for inferring biological processes linked to horns. Mismatches between volumes estimated from conical-derived formulas and true horn volume in Dall sheep O. dalli [also called thinhorn sheep (Sarasa et al., 2012)] have been mentioned previously by other authors (Heimer \& Smith, 1975). Nevertheless, these mismatches might have been underconsidered in recent studies using this type of measurement (Loehr et al., 2007, 2010). Our results also highlight the fact that length and $V_{\mathrm{c}}$ may give different biological results, as occurred in the results derived from these measurements in O. dalli (Loehr et al., 2010). Consequently, it is surprising that both measurements should produce similar results (mentioned but not fully presented) in another study of $O$. dalli (Loehr et al., 2007).

Opportunity for sexual or artificial selection requires animal trait variance (Wade \& Arnold, 1980; Arnold \& Wade, 1984). Intense debates refer to 'natural' or 'artifical' selection of horn growth (Coltman et al., 2003; Festa-Bianchet \& Lee, 2009; Loehr et al., 2010; Hedrick, 2011), although in our analysis pre-existing measurements of horn growth exhibited four to five times lower SV than true horn growth. Volume estimated as a barrel showed a SV comparable to that of true horn growth in our sample. Consequently, studies focusing on the selective pressures of horns could be greatly improved by considering alternative and appropriate proxies of true horn growth.

Horn growth measurements are not necessarily mutually comparable, just as measurements are not necessarily representative of true horn growth. Thus, results derived from pre-existing studies should be considered with caution as conflicting results in previous studies may have been caused, at least partially, by methodological differences. Further studies should reconsider the accuracy of methodologies in the analysis of tri-dimensional secondary sexual traits and biological processes linked to horns of ungulates, and maybe also to tri-dimensional weapons of other vertebrates and arthropods (Emlen, 2008), should be reappraised. Horn growth pattern is a key factor in sustainable management and conservation plans of ungulate species around the world (Coltman et al., 2003; Loehr et al., 2007; Mysterud, 2011) and, thus, our study would have numerous implications in animal conservation. 


\section{Aknowledgements}

We would like to thank the park wardens and fieldworkers from the Espacio Natural de Sierra Nevada (ENSN) and, above all, Francisco Casado Felipe and Antonio José Rodríguez Dueñas, but also Francisco J. Cano-Manuel, Antonio Rodríguez Huete, Apolo Sánchez Lao, José López Pérez, Isidro Puga González, Elías Martínez Ortiz and Manuela Cárdenas Fernández for their involvement with the study site. We are also very grateful to Paulino Fandos, Emmanuel Serrano, Georges Gonzalez and Jean Joachim for fruitful discussions, and to Ludek Bartoš and an anonymous reviewer for their comments on the paper. We would also like to thank Agnès Sarasa and Michael Lockwood for their linguistic revision. The Consejería de Medio Ambiente de Andalucía and the ENSN provided logistic support. The research activities of J.-E.G., R.C.S., M.S. and J.M.P. are partially supported by the Plan Andaluz de Investigación (RNM-118). The experiments comply with the current laws of Spain.

\section{References}

Alvarez, F. (1990). Horns and fighting in male Spanish ibex, Capra pyrenaica. J. Mammal. 71, 608-616.

Arnold, S.J. \& Wade, M.J. (1984). On the measurement of natural and sexual selection: theory. Evolution 38, 709-719.

Bartoš, L. \& Bahbouh, R. (2006). Antler size and fluctuating asymmetry in red deer (Cervus elaphus) stags and probability of becoming a harem holder in rut. Biol. J. Linn. Soc. 87, 59-68.

Bartoš, L., Bahbouh, R. \& Vach, M. (2007). Repeatability of size and fluctuating asymmetry of antler characteristics in red deer (Cervus elaphus) during ontogeny. Biol. J. Linn. Soc. 91, 215-226.

Bennett, A.T.D., Cuthill, I.C. \& Norris, K.J. (1994). Sexual selection and the mismeasure of color. Am. Nat. 144, 848-860.

Bennett, A.T.D., Cuthill, I.C., Partridge, J.C. \& Maier, E.J. (1996). Ultraviolet vision and mate choice in zebra finches. Nature 380, 433-435.

Bergeron, P., Festa-Bianchet, M., von Hardenberg, A. \& Bassano, B. (2008). Heterogeneity in male horn growth and longevity in a highly sexually dimorphic ungulate. Oikos 117, 77-82.

Bergeron, P., Grignolio, S., Apollonio, M., Shipley, B. \& Festa-Bianchet, M. (2010). Secondary sexual characters signal fighting ability and determine social rank in Alpine ibex (Capra ibex). Behav. Ecol. Sociobiol. 64, 1299-1307.

Coltman, D.W., Festa-Bianchet, M., Jorgenson, J.T. \& Strobeck, C. (2002). Age-dependent sexual selection in bighorn rams. Proc. Roy. Soc. Lond. Ser. B. 269, 165-172.

Coltman, D.W., O’Donoghue, P., Jorgenson, J.T., Hogg, J.T., Strobeck, C. \& Festa-Bianchet, M. (2003). Undesirable evolutionary consequences of trophy hunting. Nature 426, 655658.
Cornwallis, C.K. \& Uller, T. (2010). Towards an evolutionary ecology of sexual traits. Trends Ecol. Evol. 25, 145-152.

Couturier, M. (1962). Le bouquetin des Alpes (Capra aegagrus ibex ibex L.). Grenoble: Arthaud.

Croll, D.A., Clark, C.W., Acevedo, A., Tershy, B., Flores, S., Gedamke, J. \& Urban, J. (2002). Only male fin whales sing loud songs. Nature 417, 809.

Davis, E.B., Brakora, K.A. \& Lee, A.H. (2011). Evolution of ruminant headgear: a review. Proc. Roy. Soc. Lond. Ser. B. 278, 2857-2865.

Dennis, C. \& Aldhous, P. (2004). A tragedy with many players. Nature 430, 396-398.

Emlen, D.J. (2008). The evolution of animal weapons. Annu. Rev. Ecol. Evol. Syst. 39, 387-413.

Fandos, P. (1991). La cabra montés (Capra pyrenaica) en el Parque Natural de las Sierras de Cazorla Segura y las Villas. Madrid: ICONA-CSIC.

Fandos, P. (1995). Factors affecting horn growth in male Spanish ibex (Capra pyrenaica). Mammalia 59, 229-235. FestaBianchet, M. \& Lee, R. (2009). Guns, sheep, and genes: when and why trophy hunting may be a selective pressure. In Recreational hunting, conservation and rural livelihoods: science and practice: 94-107. Dickson, B., Hutton, J. \& Adams, W.M. (Eds). Oxford: Wiley-Blackwell.

Garant, D., Dodson, J.J. \& Bernatchez, L. (2003). Differential reproductive success and heritability of alternative reproductive tactics in wild Atlantic salmon (Salmo salar L.). Evolution 57, 1133-1141.

von Hardenberg, A., Bassano, B., Arranz, M.P.Z. \& Bogliani, G. (2004). Horn growth but not asymmetry heralds the onset of senescence in male Alpine ibex (Capra ibex). J. Zool. (Lond.) 263, 425-432.

Hedrick, P.W. (2011). Rapid decrease in horn size of bighorn sheep: environmental decline, inbreeding depression, or evolutionary response to trophy hunting? J. Hered. 102, 770-781.

Heimer, W.E. \& Smith, A.C. (1975). Ram horn growth and population quality: their significance to Dall sheep management in Alaska. Ak. Dept. Fish and Game. Wildl. Tech. Bull. 5, 41.

Hoefs, M. \& Nowlan, U. (1997). Comparison of horn growth in captive and free-ranging Dall's rams. J. Wildl. Mgmt. 61, 1154-1160.

Holy, T.E. \& Guo, Z. (2005). Ultrasonic songs of male mice. PLoS Biol. 3, e386.

Johnson, P.J., Kansky, R., Loveridge, A.J. \& Macdonald, D.W. (2010). Size, rarity and charisma: valuing African wildlife trophies. PLoS ONE 5, e12866.

König, R. \& Hoefs, M. (1984). Volume and density of horns of Dall rams. Bienn. Symp. North. Wild Sheep and Goat Counc. 4, 295-304.

Lailvaux, S.P., Reaney, L.T. \& Backwell, P.R.Y. (2009). Dishonest signalling of fighting ability and multiple performance traits in the fiddler crab Uca mjoebergi. Funct. Ecol. 23, 359-366. 
Lessells, C.M. \& Boag, P.T. (1987). Unrepeatable repeatabilities: a common mistake. Auk 104, 116-121.

Loehr, J., Carey, J., Hoefs, M., Suhonen, J. \& Ylönen, H. (2007). Horn growth rate and longevity: implications for natural and artificial selection in thinhorn sheep (Ovis dalli). J. Evol. Biol. 20, 818-828.

Loehr, J., Carey, J., O’Hara, R.B. \& Hik, D.S. (2010). The role of phenotypic plasticity in responses of hunted thinhorn sheep ram horn growth to changing climate conditions. J. Evol. Biol. 23, 783-790.

Luzon, M., Santiago-Moreno, J., Meana, A., Toledano-Diaz, A., Pulido-Pastor, A., Gomez-Brunet, A. \& LopezSebastian, A. (2008). Parasitism and horn quality in male Spanish ibex (Capra pyrenaica hispanica) from Andalusia based on coprological analysis and muscle biopsy. Span. J. Agric. Res. 6, 353-361.

Malo, A.F., Roldan, E.R.S., Garde, J., Soler, A.J. \& Gomendio, M. (2005). Antlers honestly advertise sperm production and quality. Proc. Roy. Soc. Lond. Ser. B. 272, 149-157.

Mysterud, A. (2011). Selective harvesting of large mammals: how often does it result in directional selection? J. Appl. Ecol. 48, 827-834.

Pérez, J.M., Granados, J.E., Sarasa, M. \& Serrano, E. (2011). Usefulness of estimated surface area of damaged skin as a proxy of mite load in the monitoring of sarcoptic mange in free-ranging populations of Iberian wild goat, Capra pyrenaica. Vet. Parasitol. 176, 258-264.

Preston, B.T., Stevenson, I.R., Pemberton, J.M., Coltman, D.W. \& Wilson, K. (2003). Overt and covert competition in a promiscuous mammal: the importance of weaponry and testes size to male reproductive success. Proc. Roy. Soc. Lond. Ser. B. 270, 633-640.

R Development Core Team (2011). R: a language and environment for statistical computing. Vienna: R Foundation for Statistical Computing (http://www.R-project.org/).

Sarasa, M., Rambozzi, L., Rossi, L., Meneguz, P.G., Serrano, E., Granados, J.E., González, F.J., Fandos, P., Soriguer,
R.C., Gonzalez, G., Joachim, J. \& Pérez, J.M. (2010a). Sarcoptes scabiei: specific immune response to sarcoptic mange in the Iberian ibex Capra pyrenaica depends on previous exposure and sex. Exp. Parasitol. 124, 265-271.

Sarasa, M., Serrano, E., Pérez, J.M., Soriguer, R.C., Gonzalez, G., Joachim, J., Fandos, P. \& Granados, J.E. (2010b). Effects of season, age, and body condition on allocation to testes mass in Iberian ibex. J. Zool. (Lond.) 281, 125-131.

Sarasa, M., Pérez, J.M., Alasaad, S., Serrano, E., Soriguer, R.C., Granados, J.E., Fandos, P., Joachim, J. \& Gonzalez, G. (2011a). Neatness depends on season, age and sex in Iberian ibex Capra pyrenaica. Behav. Ecol. 22, 1070-1078.

Sarasa, M., Serrano, E., Soriguer, R.C., Granados, J.-E., Fandos, P., Gonzalez, G., Joachim, J. \& Pérez, J.M. (2011b). Negative effect of the arthropod parasite, Sarcoptes scabiei, on testes mass in Iberian ibex, Capra pyrenaica. Vet. Parasitol. 175, 306-312.

Sarasa, M., Alasaad, S. \& Pérez, J.M. (2012). Common names of species, the curious case of Capra pyrenaica and the concomitant steps towards the 'wild-to-domestic' transformation of a flagship species and its vernacular names. Biodivers. Conserv. 21, 1-12.

Schaller, G.B. (1977). Mountain monarchs, wild sheep and goats of the Himalaya. Chicago: The University of Chicago Press.

Stearns, S.C. (1992). The evolution of life histories. Oxford: Oxford University Press.

Toïgo, C., Gaillard, J.M., Festa-Bianchet, M., Largo, E., Michallet, J. \& Maillard, D. (2007). Sex- and age-specific survival of the highly dimorphic Alpine ibex: evidence for a conservative life-history tactic. J. Anim. Ecol. 76, 679-686.

Wade, M.J. \& Arnold, S.J. (1980). The intensity of sexual selection in relation to male sexual behaviour, female choice, and sperm precedence. Anim. Behav. 28, 446-461.

Wood, S.N. (2006). Generalized additive models, an introduction with R. Boca Raton: Chapman \& Hall/CRC. 